\title{
Extremal quasiconformality vs rational approximation
}

\author{
SAmuel L. Krushkal
}

(Presented by V. Gutlyanskiน)

The paper is devoted to the 100th anniversary of Georgii Dmitrievich Suvorov, my first university adviser and teacher. He was an outstanding mathematician and a widely talented, extremely great human being.

\begin{abstract}
We show that on most of the hyperbolic simply connected domains the weighty bounded rational approximation in a natural sup norm is possible only for a very sparse set of holomorphic functions (in contrast to integral approximation). The obstructions are caused by the features of extremal quasiconformality.
\end{abstract}

2010 MSC. Primary: 30C62, 30C75, 30E10; Secondary: 30F45, 30F60, $32 \mathrm{G} 15$.

Key words and phrases. Rational approximation, holomorphic function, quasiconformal maps, quasicircles, universal Teichmüller space, Schwarzian derivative, Strebel point, Grunsky coefficients.

\section{Results}

This paper gives a link of geometric function theory to weighty bounded rational approximation of holomorphic functions in sup norms and shows how the intrinsic features of extremal quasiconformal maps and universal Teichmüller space provide strong obstructions to such an approximation. The situation is completely different from the integral approximation.

\subsection{Introductory remarks}

The classical directions in rational interpolation of holomorphic functions investigated by many authors concern mainly the uniform interpolation of functions holomorphic in the inner points of the closed set 
$X$ on the Riemann sphere $\widehat{\mathbb{C}}=\mathbb{C} \cup\{\infty\}$ and continuous on $X$ by rational functions with poles off $X$ and its combination with interpolation (see, e.g., [26]). The second approach was originated by Walsh (see $[2,25]$ ) and has recently been extended in [11] to the functional space $A^{-\infty}=\bigcup_{q>0} A^{-q}$ over the Dini domains (with topology of the inductive limit), where $A^{-q}(D)$ is the Banach space of holomorphic functions in a domain $D$ with norm $\|f\|=\sup _{D} \delta_{D}(z)|f(z)|$; here $\delta_{D}(z)=\operatorname{dist}(z, \partial D)$ denotes the Euclidean distance from the point $z \in D$ to the boundary.

More generally, one considers a space $\mathcal{F}$ of holomorphic functions $f$ in a domain $D \subset \widehat{\mathbb{C}}$. For any given collections of points

$$
A_{n}=\left\{a_{n j}\right\}_{j=0}^{n} \subset D, \quad B_{n}=\left\{b_{n j}\right\}_{j=1}^{n} \subset \widehat{\mathbb{C}} \backslash D \quad(n=1,2, \ldots),
$$

there exists a unique rational function $r_{n, f}$ of degree $n$, with poles at $B_{n}$, interpolating to $f$ at $A_{n}$, counting multiplicities. The problem is to select these collections $A_{n}$ and $B_{n}$ so that for all $f$ the interpolants $r_{n, f}$ converge to $f$ in the topology of $\mathcal{F}$.

It is established in [11] (by sweeping out appropriate measures and application of the potential methods) that for $f \in A^{-q}(D), q>0$, the interpolants $r_{n, f}$ are convergent (under appropriate conditions) to $f$ in $A^{-q^{\prime}}$ norm, where $q^{\prime}(q) \gg q$.

\subsection{Weakened rational approximation in sup norm}

Let $L$ be an oriented quasiconformal Jordan curve (quasicircle) on the Riemann sphere $\widehat{\mathbb{C}}=\mathbb{C} \cup\{\infty\}$ with the interior and exterior domains $D$ and $D^{*}$, and let $p \geq 2$ be an integer. Denote by $\lambda_{D}(z)|d z|$ the hyperbolic metric of $D$ of Gaussian curvature -4 and consider the Banach spaces $A_{p}(D), \mathbf{B}_{p}(D)$ of holomorphic functions (quadratic differentials) $\varphi$ with norms

$$
\|\varphi\|_{\mathbf{B}_{p}}=\sup _{D} \lambda_{D}(z)^{-p}|\varphi(z)|, \quad\|\varphi\|_{A_{p}}=\iint_{D} \lambda_{D}(z)^{2-p}|\varphi(z)| d x d y,
$$

respectively; due to [5], the space $\mathbf{B}_{p}$ is dual to $A_{p}$.

Note that for simply connected domains (with more than one boundary points) not containing inside the infinite point,

$$
\frac{1}{4} \leq \lambda_{D}(z) \delta_{D}(z) \leq 1
$$

where the right hand inequality follows from the Schwarz lemma and the left from Koebe's $\frac{1}{4}$ theorem (so the spaces $A^{-p}$ mentioned above are obtained by renormalization of $\mathbf{B}_{p}$ ).

We also shall use the notations 


$$
\begin{gathered}
\mathbb{D}=\{z:|z|<1\}, \quad \mathbb{D}^{*}=\{z \in \widehat{\mathbb{C}}:|z|>1\} \\
\mathbb{H}=\{z: \operatorname{Im} z>0\}, \mathbb{H}^{*}=\{z: \operatorname{Im} z<0\}
\end{gathered}
$$

We start with the following general

Theorem 1. Let $D \subset \widehat{\mathbb{C}}$ be a domain with quasiconformal boundary $L$. Then for any function $f \in \mathbf{B}_{p}(D)$ there exists a sequence of rational functions with poles of order two on $L$ of the form

$$
r_{n}(z)=\sum_{1}^{n} \frac{c_{j}}{\left(z-a_{j}\right)^{2}}, \quad \sum_{1}^{n}\left|c_{j}\right|>0
$$

such that $\lim _{n \rightarrow \infty}\left\|r_{n}-\varphi\right\|_{\mathbf{B}_{p+1}(D)}=0$.

In the case of the half-plane (or disk), this theorem is strengthened as follows.

Theorem 2. For any $\varphi \in \mathbf{B}_{p}(\mathbb{H})$ there exists a sequence of rational functions (2) with real poles $a_{j}$ and real coefficients $c_{j}$, convergent to $\varphi$ in $\mathbf{B}_{p+1}(\mathbb{H})$.

One can see from the proof of Theorem 1 that the weight exponent $p+1$ is not sharp; though it is not clear whether this exponent can be replaces by $p+\epsilon(p)$ with $0<\epsilon(p)<1$.

The limit case $\epsilon=0$ has intrinsic interest. Then the assertion on convergence fails for $\epsilon=0$, because the space $\mathbf{B}_{2}$ of bounded holomorphic quadratic differentials $\varphi d z^{2}$ is not separable for any Riemann surface of infinite genus, and similar for all $\mathbf{B}_{p}$.

This was established by functional-analytic methods but can be established also from geometric features generated by Thurston's theorem on existence of uncountable many conformally rigid domains (see [3,24]). Such domains correspond to the isolated points of the set $\mathbf{U} \backslash \mathbf{T}$ in $\mathbf{B}_{2}(\mathbb{H})$, where $\mathbf{U}$ denotes the set of the Schwarzian derivatives

$$
S_{w}(z)=\left(\frac{w^{\prime \prime}(z)}{w^{\prime}(z)}\right)^{\prime}-\frac{1}{2}\left(\frac{w^{\prime \prime}(z)}{w^{\prime}(z)}\right)^{2}
$$

of all univalent functions on $\mathbb{H}$ and $\mathbf{T}$ is the universal Teichmüller space modeled by a bounded domain in $\mathbf{B}_{2}(\mathbb{H})$ (formed by $f$ having quasiconformal extension to $\widehat{\mathbb{C}}$ ).

Note also that any $\varphi \in \mathbf{B}_{2}(D)$ can be regarded as the Schwarzian of a locally univalent function in $D$ and determines this function up to a Moebius transformation of $\widehat{\mathbb{C}}$. 


\subsection{Main theorems}

Our aim is to show that on most of the hyperbolic simply connected domains the rational approximation in $\mathbf{B}_{2}$ is possible only for a very sparse subset of functions.

Theorem 3. For any simply connected domain $D \subset \widehat{\mathbb{C}}$ with quasiconformal boundary $L$, whose conformal mapping function $g_{D}: \mathbb{H} \rightarrow D$ satisfies $\left\|S_{g_{D}}\right\|_{\mathbf{B}_{2}}<1 / 2$, the set of functions $\varphi \in \mathbf{B}_{2}(D)$ approximated in $\mathbf{B}_{2}$ norm by general rational functions with poles of order two on $L$,

$$
r_{n}(z)=\sum_{1}^{n} \frac{c_{j}}{\left(z-a_{j}\right)^{2}}+\sum_{1}^{n} \frac{c_{j}^{\prime}}{z-a_{j}}, \quad \sum_{1}^{n}\left|c_{j}\right|>0
$$

is nonwhere dense in the space $\mathbf{B}_{2}(D)$.

This theorem is a consequence of some deep results concerning the extremal maps and the Grunsky operator given by

Theorem 4. There exists a constant $c_{0}>0$ such that for any simply connected domain $D \subset \widehat{\mathbb{C}}$ with quasiconformal boundary $L$ and such that $\left\|S_{g_{D}}\right\|_{\mathbf{B}_{2}}<1 / 2$, and for any rational function $r_{n}$ with poles of order two on $L$ of the form (3) and norm $\left\|r_{n}\right\|_{\mathbf{B}_{2}(D)}<c_{0}$, we have the equalities

$$
\varkappa_{D}(w)=k(w)=\left\|r_{n}\right\|_{\mathbf{B}_{2}(D)},
$$

where $\varkappa_{D}(w)$ and $k(w)$ denote the Grunsky and Teichmüller norms of (appropriately normalized) univalent solution $w: D \rightarrow \widehat{\mathbb{C}}$ of the Schwarzian equation $S_{f}=r_{n}$.

Note that the indicated constant $c_{0}$ does not depend on $D$. In the case of a disk (half-plane), one can take $c_{0}=1 / 2$.

Theorem 4 shows that all Schwarzians $\varphi=r_{n}$ with $\left\|r_{n}\right\|_{\mathbf{B}_{2}}<c_{0}$ are not Strebel points in the universal Teichmüller space $\mathbf{T}$ (in other words, the conformal maps $f$ with $S_{f}=r_{n}$ do not have Teichmüller extremal extensions onto the complementary domain $\left.D^{*}=\widehat{\mathbb{C}} \backslash \bar{D}\right)$. Hence, such points cannot be dense in $\mathbf{B}_{2}(D)$.

Note also that, in view of the first equality in (4), the dilatation $k(f)$ is attained on the squares of holomorphic abelian differentials $\omega d z$ on $D$.

Theorem 3 is an immediate consequence of the equalities (4) in view of either of two basic results on openness and density in the universal 
Teichmüller space $\mathbf{T}$ : the result of [14] for points with non-equal Teichmüller and Grunsky norms and Lakic's result [19] on the density of Strebel points (in arbitrary Teichmüller space).

Indeed, it suffices to establish the assertion of Theorem 3 for $\varphi \in$ $\mathbf{B}_{2}(D)$ with $\|\varphi\|<c_{0}$. Both Teichmüller and Grunsky norms are continuous on the universal Teichmüller space $\mathbf{T}$ modelled as a bounded domain in $\mathbf{B}_{2}$ containing the origin. Hence, the equality (4) is preserving also for the limit function $f=\lim r_{n}$ of any sequence of rational functions in $\mathbf{B}_{2}$.

But this equality implies that any $f \in \mathbf{B}_{2}$ with sufficiently small $\|f\|$ generates the Beltrami coefficient

$$
\mu(z)=\frac{1}{2}|z-\bar{z}|^{2} f \circ g_{D}(\bar{z}),
$$

where $g_{D}$ is a conformal map of $\mathbb{H}$ onto domain $D$ (called harmonic), and this coefficient is extremal in its equivalence class. On the other side, it is not of Teichmüller type.

The latter is impossible in view of the indicated above openness results.

\subsection{Generalization of Theorems 3 and 4}

One can see from the proof of Theorem 4 in Section 5 that actually its arguments are valid for an arbitrary meromorphic function $\varphi$ on $\mathbb{C}$ having poles of order two which are located on a quasicircle $L$ passing through the infinite point and accumulate to this point. This gives the following extension of the above theorems.

Theorem 5. For any simply connected domain $D \in \widehat{\mathbb{C}}$ with quasiconformal boundary $L$ passing through the infinite point whose conformal mapping function satisfies $\left\|S_{g_{D}}\right\|_{\mathbf{B}_{2}}<1 / 2$, the subspace $\mathcal{M}_{2}$ in $\mathbf{B}_{2}(D)$ formed by meromorphic functions $\varphi$ on $\mathbb{C}$ with poles of order two, which are located on $L$ and accumulate to $\infty$, is nonwhere dense in the space $\mathbf{B}_{2}(D)$.

If $\|\varphi\|_{\mathbf{B}_{2}(D)}$ is sufficiently small, so that the Schwarzian equation $S_{w}=\varphi$ has a univalent solution $w(z)$ on $D$, then

$$
\varkappa_{D}(w)=k(w)=\|\varphi\|_{\mathbf{B}_{2}(D)} .
$$

\section{Proof of Theorem 1}

First note that $\left.A_{p}(D) \subset \mathbf{B}_{p} D\right)$, and for any $\varphi \in A_{p}(D)$,

$$
\|\varphi\|_{\mathbf{B}_{p}} \leq \frac{4}{\pi}\|\varphi\|_{A_{p}}
$$


Indeed, since both these norms are conformally invariant, it suffices to verify (5) for $D=\mathbb{H}$. Then $\lambda_{D}(z)=1 /(2 y)$, and applying the mean inequality for holomorphic functions, one gets

$$
|f(z)| \leq \frac{1}{\pi y^{2}} \iint_{|z-\zeta| \leq y}|f(\zeta)| d \xi d \eta \leq \frac{(2 y)^{2-p}}{\pi y^{2}} \iint_{|z-\zeta| \leq y}(2 \eta)^{2-p} d \xi d \eta
$$

(here $\zeta=\xi+i \eta, z \in D, \eta \leq 2 y$ ), which yields (5). It follows also that for any $\varphi \in A_{p}(D)$,

$$
\lim _{\rho \rightarrow 0} \sup _{\delta_{D}(z) \geq \rho} \lambda_{D}^{p}(z)|\varphi(z)|=0 .
$$

Without loss of generality, one can assume that the boundary curve $L \ni \infty$ and $0 \in D$. For any $\varphi \in \mathbf{B}_{p}(D)$,

$$
|\varphi(z)| \leq\|\varphi\|_{B_{p}} \lambda_{D}(z)^{-p} \asymp\|\varphi\|_{B_{p}} \delta_{D}(z)^{-p} ;
$$

hence it belongs to $A_{p+1}$ and its integral

$$
I_{\varphi}(z)=\int_{0}^{z} \varphi(\zeta) d \zeta
$$

belongs to $A_{p}$.

By the Bers approximation theorem [4], there exists a sequence of rational functions $\widetilde{r}_{j}(z)$ with simple poles on $L$ and no other singularities, such that ${ }^{1}$

$$
\lim _{n \rightarrow \infty}\left\|\widetilde{r}_{j}-I_{\varphi}\right\|_{A_{p}}=0
$$

Further, due to [5], for every $\psi \in \mathbf{B}_{p}(D)$ the following reproducing formula is valid:

$$
\psi(z)=-\frac{2 p-1}{\pi} \iint_{D} \frac{\left(\zeta-h(\zeta)^{2 p-2}(\partial h(\zeta) / \partial \bar{\zeta})\right) \psi(h(\zeta))}{(\zeta-z)^{2 p}} d \xi d \eta
$$

here $\zeta \mapsto h(\zeta)$ is a quasiconformal reflection with respect to the quasicircle $L=\partial D$ (i.e., orientation reversing quasiconformal automorphism of $\widehat{\mathbb{C}}$ mapping $D$ onto its complementary domain and leaving fixed all points of $L$ ) which is uniformly bilipschizian on $\mathbb{C}$, i.e., for all points $z_{1}, z_{2} \in \mathbb{C}$ the inequality

$$
c_{0}^{-1}\left|z_{1}-z_{2}\right| \leq\left|h\left(z_{1}\right)-h\left(z_{2}\right)\right| \leq c_{0}\left|z_{1}-z_{2}\right|
$$

\footnotetext{
${ }^{1}$ This theorem is proved in [4] for the integrable holomorphic functions $f \in A_{2}$; the proof for the weighted spaces $A_{p}$ can be done along the same lines, see [12].
} 
holds with some constant $c_{0}>1$. Moreover, the numerator

$$
\nu_{\psi}(\zeta)=-\frac{2 p-1}{\pi}(\zeta-h(\zeta))^{2 p-2} \frac{\partial h(\zeta)}{\partial \bar{\zeta}}
$$

is estimated uniformly by

$$
\left|\nu_{\psi}(\zeta)\right| \leq c\left(c_{0}\right)\|\psi\|_{\mathbf{B}_{2}(D)} \lambda_{D}(\zeta)^{2-p}
$$

Applying (8) to $\psi=\widetilde{r}_{j}-I_{\varphi}$ and differentiating both sides in $z$, one obtains from (7) that $r_{j}=\widetilde{r}_{j}^{\prime}$ are convergent to $\varphi$ in $\mathbf{B}_{p+1}(D)$, completing the proof of the theorem.

\section{Proof of Theorem 2}

We apply the following result on integral approximation in the unit disk given in [12] improving for the disk the Bers approximation theorem mentioned above and also related to the theory of extremal quasiconformal maps.

Proposition 1. Let $p$ and $m$ be two integers such that $p \geq 2$ and $m \geq 1$. Then for any $\psi \in A_{p}(\mathbb{D})$, there exists a sequence of rational functions $\widetilde{r}_{j}$ which have only simple poles on the unit circle $S^{1}$ and satisfy the condition $\operatorname{Im}\left[\zeta^{m} \widetilde{r}_{j}(\zeta)\right]=0$ on $S^{1}$ (outside of the poles of $\widetilde{r}_{j}$ ), such that

$$
\lim _{j \rightarrow \infty}\left\|\widetilde{r}_{j}-\psi\right\|_{A_{p}}=0
$$

For even $m$ and $\zeta=e^{i \theta} \in S^{1}$, we have

$$
\operatorname{Im}\left[\widetilde{r}_{j}(\zeta) d \zeta^{m}\right]=(-1)^{m / 2} \operatorname{Im}\left[\zeta^{m} \widetilde{r}_{j}(\zeta)\right] d \theta^{m}
$$

thus the above proposition can be reformulated as follows.

Proposition 2. For any function $\psi \in A_{p}(\mathbb{D})$, there exists a sequence of rational functions $\widetilde{r}_{j}$ which have only simple poles on the circle $S^{1}$ and satisfy the condition $\operatorname{Im}\left[\widetilde{r}_{j}(\zeta) d \zeta^{2 m}\right]=0$ on $S^{1}$, such that $\lim _{j \rightarrow \infty} \| \widetilde{r}_{j}-$ $\psi \|_{A_{p}}=0$.

Take $m=p$ and, applying the fractional linear map $\sigma(z)=(z-$ $i) /(z+i)$ of the upper half-plane onto the unit disk, approximate similar to Theorem 1 the integrated functions

$$
\sigma_{*} I_{\psi}=\left(I_{\psi} \circ \sigma\right)\left(\sigma^{\prime}\right)^{4 m-2}
$$


by the corresponding rational functions

$$
\sigma_{*} \widetilde{r}_{j}=\left(\widetilde{r}_{j} \circ \sigma\right)\left(\sigma^{\prime}\right)^{4 m-2} \in A_{p+1}(\mathbb{H})
$$

having real poles and coefficients in view of (9). Now applying the reproducing formula for the upper half-plane,

$$
\psi(z)=\frac{2 p-1}{\pi} \iint_{D} \frac{(\zeta-\bar{\zeta})^{2 p-2} \psi(\bar{\zeta})}{(\zeta-z)^{2 p}} d \xi d \eta
$$

one straightforwardly obtains the conclusion of Theorem 2 .

\section{Backgrounds of Theorem 3}

As was mentioned, Theorem 3 relies on some intrinsic features of extremal quasiconformal maps and the Grunsky operator. For convenience, we briefly describe here these underlying results.

\subsection{Extremal quasiconformality}

Let $L$ be a quasicircle passing through the points $0,1, \infty$ which is the common boundary of two domains $D$ and $D^{*}$. Take the unit ball of Beltrami coefficients supported on $D^{*}$,

$$
\operatorname{Belt}\left(D^{*}\right)_{1}=\left\{\mu \in L_{\infty}(\mathbb{C}): \mu \mid D=0 \quad\|\mu\|_{\infty}<1\right\}
$$

and consider the corresponding quasiconformal automorphisms $w^{\mu}(z)$ of the sphere $\widehat{\mathbb{C}}$ satisfying on $\mathbb{C}$ the Beltrami equation $\bar{\partial} w=\mu \partial w$ preserving the points $0,1, \infty$ fixed. We call the quantity $k(w)=\left\|\mu_{w}\right\|_{\infty}$ the dilatation of the map $w$.

Take the equivalence classes $[\mu]$ and $\left[w^{\mu}\right]$ letting the coefficients $\mu_{1}$ and $\mu_{2}$ from $\operatorname{Belt}\left(D^{*}\right)_{1}$ be equivalent if the corresponding maps $w^{\mu_{1}}$ and $w^{\mu_{2}}$ coincide on $L$ (and hence on $\bar{D}$ ). These classes are in one-to-one correspondence with the Schwarzians $S_{w^{\mu}}$ on $D$ which fill a bounded domain in the space $\mathbf{B}_{2}(D)$ modelling the universal Teichmüller space $\mathbf{T}=\mathbf{T}(D)$ with the base point $D$. The quotient map

$$
\phi_{\mathbf{T}}: \operatorname{Belt}\left(D^{*}\right)_{1} \rightarrow \mathbf{T}, \quad \phi_{\mathbf{T}}(\mu)=S_{w^{\mu}}
$$

is holomorphic (as the map from $L_{\infty}\left(D^{*}\right)$ to $\mathbf{B}_{2}(D)$ ). Its intrinsic $T e$ ichmüller metric is defined by $\tau_{\mathbf{T}}\left(\phi_{\mathbf{T}}(\mu), \phi_{\mathbf{T}}(\nu)\right)=\frac{1}{2} \inf \left\{\log K\left(w^{\mu_{*}} \circ\left(w^{\nu_{*}}\right)^{-1}\right): \mu_{*} \in \phi_{\mathbf{T}}(\mu), \nu_{*} \in \phi_{\mathbf{T}}(\nu)\right\}$, 
It is the integral form of the infinitesimal Finsler metric

$$
F_{\mathbf{T}}\left(\phi_{\mathbf{T}}(\mu), \phi_{\mathbf{T}}^{\prime}(\mu) \nu\right)=\inf \left\{\left\|\nu_{*} /\left(1-|\mu|^{2}\right)\right\|_{\infty}: \quad \phi_{\mathbf{T}}^{\prime}(\mu) \nu_{*}=\phi_{\mathbf{T}}^{\prime}(\mu) \nu\right\}
$$

on the tangent bundle $\mathcal{T} \mathbf{T}$ of $\mathbf{T}$, which is locally Lipschitzian.

We call the Beltrami coefficient $\mu \in \operatorname{Belt}\left(D^{*}\right)_{1}$ extremal (in its class) if

$$
\|\mu\|_{\infty}=\inf \left\{\|\nu\|_{\infty}: \quad \phi_{\mathbf{T}}(\nu)=\phi_{\mathbf{T}}(\mu)\right\}
$$

and call $\mu$ infinitesimally extremal if

$$
\|\mu\|_{\infty}=\inf \left\{\|\nu\|_{\infty}: \quad \nu \in L_{\infty}\left(D^{*}\right), \quad \phi_{\mathbf{T}}^{\prime}(\mathbf{0}) \nu=\phi_{\mathbf{T}}^{\prime}(\mathbf{0}) \mu\right\} .
$$

Any infinitesimally extremal Beltrami coefficient $\mu$ is globally extremal (and vice versa), and by the basic Hamilton-Krushkal-Reich-Strebel theorem the extremality of $\mu$ is equivalent to the equality

$$
\|\mu\|_{\infty}=\inf \left\{\left|<\mu, \psi>_{D^{*}}\right|: \quad \psi \in A_{2}\left(D^{*}\right):\|\psi\|=1\right\}
$$

(where $A_{2}\left(D^{*}\right)$ is the subspace of $L_{1}\left(D^{*}\right)$ formed by holomorphic functions on $D^{*}$ ) and the pairing

$\langle\mu, \psi\rangle_{D^{*}}=\iint_{D^{*}} \mu(z) \psi(z) d x d y, \quad \mu \in L_{\infty}\left(D^{*}\right), \psi \in L_{1}\left(D^{*}\right)(z=x+i y)$.

Let $w_{0}:=w^{\mu_{0}}$ be an extremal representative of its class $\left[w_{0}\right]$ with dilatation

$$
k\left(w_{0}\right)=\left\|\mu_{0}\right\|_{\infty}=\inf \left\{k\left(w^{\mu}\right): w^{\mu}\left|L=w_{0}\right| L\right\}
$$

and assume that there exists in this class a quasiconformal map $w_{1}$ whose Beltrami coefficient $\mu_{A_{1}}$ satisfies the inequality ess $\sup _{A_{r}}\left|\mu_{w_{1}}(z)\right|<k\left(w_{0}\right)$ in some ring domain $\mathcal{R}=D^{*} \backslash G$ complement to a domain $G \supset D^{*}$. Any such $w_{1}$ is called the frame map for the class $\left[w_{0}\right]$, and the corresponding point in the universal Teichmüller space $\mathbf{T}$ is called the Strebel point.

These points have the following important properties.

Proposition 3. (i) If a class [f] has a frame map, then the extremal map $f_{0}$ in this class (minimizing the dilatation $\|\mu\|_{\infty}$ ) is unique and either a conformal or a Teichmüller map with Beltrami coefficient $\mu_{0}=k\left|\psi_{0}\right| / \psi_{0}$ on $D^{*}$, defined by an integrable holomorphic quadratic differential $\psi_{0}$ on $D^{*}$ and a constant $k \in(0,1)[23]$.

(ii) The set of Strebel points is open and dense in $\mathbf{T}[8,19]$. 
The first assertion holds, for example, for asymptotically conformal (hence for all smooth) curves $L$. Similar results hold also for arbitrary Riemann surfaces (cf. $[7,8]$ ).

The boundary dilatation $H(f)$ admits also a local version $H_{p}(f)$ involving the Beltrami coefficients supported in the neighborhoods of a boundary point $p \in \partial D$. Moreover (see, e.g., [8, Ch. 17]), $H(f)=$ $\sup _{p \in \partial D} H_{p}(f)$, and the points with $H_{p}(f)=H(f)$ are called substantial for $f$ and for its equivalence class.

\subsection{The Grunsky-Milin inequalities}

Let $D^{*} \ni \infty$ be a simply connected domain with quasiconformal boundary and $\Sigma^{0}\left(D^{*}\right)$ denote the class of univalent $\widehat{\mathbb{C}}$-holomorphic functions in $D^{*}$ with expansions $f(z)=z+b_{0}+b_{1} z^{-1}+\ldots$ near $z=\infty$ admitting quasiconformal extensions to $\widehat{\mathbb{C}}$. Their Grunsky-Milin coefficients $\alpha_{m n}$ are defined from the expansion

$$
-\log \frac{f(z)-f(\zeta)}{z-\zeta}=\sum_{m, n=1}^{\infty} \frac{\alpha_{m n}}{\chi(z)^{m} \chi(\zeta)^{n}},
$$

choosing the branch of the logarithmic function which vanishes as $z=$ $\zeta \rightarrow \infty$. Here $\chi$ denotes a conformal map of $D^{*}$ onto the disk $\mathbb{D}^{*}$ so that $\chi(\infty)=\infty, \chi^{\prime}(\infty)>0$.

Each coefficient $\alpha_{m n}(f)$ in (10) is a polynomial of a finite number of the initial coefficients $b_{1}, b_{2}, \ldots, b_{m+n-1}$ of $f$; hence it depends holomorphically on Beltrami coefficients of extensions of $f$ as well as on the Schwarzian derivatives $S_{f} \in \mathbf{B}_{2}\left(D^{*}\right)$.

A theorem of Milin extending the Grunsky univalence criterion for the disk $\mathbb{D}^{*}$ states that a holomorphic function $f(z)=z+$ const $+O\left(z^{-1}\right)$ in a neighborhood of $z=\infty$ can be continued to a univalent function in the whole domain $D^{*}$ if and only if the coefficients $\alpha_{m n}$ satisfy the inequality

$$
\left|\sum_{m, n=1}^{\infty} \sqrt{m n} \alpha_{m n} x_{m} x_{n}\right| \leq 1
$$

for any point $\mathbf{x}=\left(x_{n}\right)$ from the unit sphere $S\left(l^{2}\right)$ of the Hilbert space of sequences $\mathbf{x}=\left(x_{n}\right)$ with $\|\mathbf{x}\|^{2}=\sum_{1}^{\infty}\left|x_{n}\right|^{2}$ (cf. [10,20,22]). We call the quantity

$$
\varkappa_{D^{*}}(f)=\sup \left\{\left|\sum_{m, n=1}^{\infty} \sqrt{m n} \alpha_{m n} x_{m} x_{n}\right|: \mathbf{x}=\left(x_{n}\right) \in S\left(l^{2}\right)\right\}
$$


the Grunsky norm of $f$. The inequality $\varkappa_{D^{*}}(f) \leq 1$ is necessary and sufficient for univalence of $f$ in $D^{*}$ (see $\left.[10,20,22]\right)$. In the canonical case $D^{*}=\mathbb{D}^{*}$, we have the classical Grunsky coefficients.

Consider the set

$$
A_{2}^{2}(D)=\left\{\psi \in A_{2}(D): \psi=\omega^{2}\right\}
$$

consisting of the integrable holomorphic functions on $D$ having only zeros of even order and put

$$
\alpha_{D}(f)=\sup \left\{\left|\left\langle\mu_{0}, \psi\right\rangle_{D}\right|: \psi \in A_{2}^{2},\|\psi\|_{A_{2}(D)}=1\right\} .
$$

The following proposition from [16] completely describes the relation between the Grunsky and Teichmüller norms (more special results were obtained in $[13,18])$.

Proposition 4. For all $f \in \Sigma^{0}\left(D^{*}\right)$,

$$
\varkappa_{D^{*}}(f) \leq k \frac{k+\alpha_{D}(f)}{1+\alpha_{D}(f) k}, \quad k=k(f),
$$

and $\varkappa_{D^{*}}(f)<k$ unless

$$
\alpha_{D}(f)=\left\|\mu_{0}\right\|_{\infty},
$$

where $\mu_{0}$ is an extremal Beltrami coefficient in the equivalence class $[f]$. The last equality is equivalent to $\varkappa_{D^{*}}(f)=k(f)$.

If $\varkappa_{D^{*}}(f)=k(f)$ and the class of $[f]$ is a Strebel point, then $\mu_{0}$ is necessarily of the form

$$
\mu_{0}=\left\|\mu_{0}\right\|_{\infty}\left|\psi_{0}\right| / \psi_{0} \text { with } \quad \psi_{0} \in A_{2}^{2}(D) .
$$

Note that geometrically (11) means the equality of the Carathéodory and Teichmüller distances on the geodesic disk $\left\{\phi_{\mathbf{T}}\left(t \mu_{0} /\left\|\mu_{0}\right\|\right): t \in \mathbb{D}\right\}$ in the universal Teichmüller space $\mathbf{T}$.

\section{Proof of Theorem 4}

We first prove this theorem for $D=\mathbb{H}$ (and hence for the disk). In this canonical case, one gets a somewhat stronger result; moreover, the arguments are simpler and illustrate all underlying features. Now the poles of $r_{n}$ are real, and $\lambda_{\mathbb{H}}(z)=1 /|z-\bar{z}|=1 /(2 y)$.

The assertion of the theorem follows from the next two lemmas. The first lemma ensures the existence for any $r_{n} \in \mathbf{B}_{2}(D)$ of a sequence of points $z_{n} \in D$ convergent to a boundary point $a_{0}$ on which the supremum 
of $\lambda_{D}^{-2}\left|r_{n}\right|$ is attained (such $a_{0}$ can be distinct from the poles of $r_{n}$ ). The second lemma yields that this $a_{0}$ must be an essential point for $r_{n}$, and therefore this function represents a non-Strebel point.

Of course, all this is valid to much more general functions from $\mathbf{B}_{2}(D)$. A special case (the convex hull of fractions $1 /(z-a)^{2}$ with real $a$ ) was considered in [15].

Lemma 1. For any simply connected domain $D \subset \widehat{\mathbb{C}}$ with quasiconformal boundary $L$ and any rational function $r_{n}$ with poles of order two on $L$ of the form (3),

$$
\left\|r_{n}\right\|_{\mathbf{B}_{2}(D)}=\limsup _{z \rightarrow L} \lambda_{D}(z)^{-2}\left|r_{n}(z)\right| .
$$

So, there is a boundary point $z_{0}$ at which the maximal value of $\lambda_{D}(z)^{-2}\left|r_{n}(z)\right|$ on $\bar{D}$ is attained.

Proof. Consider first the case $D=\mathbb{D}$, and let $r_{n} \in \mathbf{B}_{2}(\mathbb{D})$ satisfy

$$
\limsup _{|z| \rightarrow 1}\left(1-|z|^{2}\right)^{2}\left|r_{n}(z)\right|<\sup _{z \in \mathbb{D}}\left(1-|z|^{2}\right)^{2}\left|r_{n}(z)\right|,
$$

i.e., the polyanalytic function

$$
F(z)=(1-z \bar{z})^{2}\left|r_{n}(z)\right|
$$

with $F(0)=r_{n}(0)$ attains its maximal value on $\overline{\mathbb{D}}$ at some inner point $z_{0} \in \mathbb{D}$. Applying, if needed, the conformal automorphism

$$
z \mapsto\left(z-z_{0}\right) /\left(1-\bar{z}_{0} z\right)
$$

of $\mathbb{D}$, one reduces the proof to the case $z_{0}=0$.

If $r_{n}^{\prime}(0)=a \neq 0$, then $r_{n}(z)=r_{n}(0)+a z+\ldots$, and hence, for $z=\rho e^{i \theta}$ and small $\rho>0$,

$$
\max _{\theta}\left|r_{n}\left(\rho e^{i \theta}\right)\right|=\left|r_{n}(0)\right|+|a| \rho+O\left(\rho^{2}\right) .
$$

This yields

$$
\max _{\theta}\left|F\left(\rho e^{i \theta}\right)\right|=|F(0)|+|a| \rho+O\left(\rho^{2}\right)>|F(0)|, \quad \rho \rightarrow 0,
$$

which contradicts the maximality of $|F(z)|$ at $z=0$. So, for such rational functions $r_{n}$, the inequality (13) can never occur, and

$$
\limsup _{|z| \rightarrow 1}\left(1-|z|^{2}\right)^{2}\left|r_{n}(z)\right|=\sup _{z \in \mathbb{D}}\left(1-|z|^{2}\right)^{2}\left|r_{n}(z)\right|=\left\|r_{n}\right\|_{\mathbf{B}_{2}(\mathbb{D})} .
$$


If $r_{n}^{\prime}(0)=0$, we approximate this function by rational $r_{n, \varepsilon}$ with the same poles $a_{k} \in \partial \mathbb{D}$, replacing one of the coefficients $c_{k}$ by $c_{k}+\varepsilon$ so that $r_{n, \varepsilon}^{\prime}(0) \neq 0$.

Since at the point $z_{0}$, where the function

$$
F_{\varepsilon}(z)=(1-z \bar{z})^{2}\left|r_{n, \varepsilon}(z)\right|
$$

attains its maximal value, this value is positive, one can define in a neighborhood of $z_{0}$ a single valued branch $g_{n, \varepsilon}(z)=\sqrt{r_{n, \varepsilon}(z)}$, and in this neighborhood

$$
F_{\varepsilon}(z)=(1-z \bar{z})^{2} g_{n, \varepsilon}(z) \overline{g_{n, \varepsilon}(z)} \text {. }
$$

Noting that both partial derivatives $\partial_{z} F_{\varepsilon}(z), \partial_{\bar{z}} F_{\varepsilon}(z)$ vanish at $z_{0}$ and

$$
\partial_{z} F_{\varepsilon}(z)=-2(1-z \bar{z}) \bar{z} g_{n, \varepsilon}(z) \overline{g_{n, \varepsilon}(z)}+(1-z \bar{z})^{2} \overline{g_{n, \varepsilon}(z)} g_{n, \varepsilon}^{\prime}(z)
$$

one obtains

$$
-2 \bar{z}_{0} g_{n, \varepsilon}\left(z_{0}\right)+\left(1-z_{0} \bar{z}_{0}\right) g_{n, \varepsilon}^{\prime}\left(z_{0}\right)=0
$$

and therefore,

$$
g_{n, \varepsilon}^{\prime}\left(z_{0}\right)=-\frac{2 \bar{z}_{0}}{1-z_{0} \bar{z}_{0}} g_{n, \varepsilon}(z) \neq 0 .
$$

This yields, in the same manner as above, that every such $r_{n, \varepsilon}$ satisfies the equality (12). Since this equality remains valid in the limit as $\varepsilon \rightarrow 0$, the assertion of Lemma for $D=\mathbb{D}$ is established.

The case of the generic quasidisk $D$ is reduced to the above one, taking a conformal map $\chi_{D}$ function of $D$ onto the disk $\mathbb{D}$ with $\chi_{D}\left(z_{0}\right)=0$ and applying the above arguments to functions $r_{n} \circ \chi$, which have the same properties as $r_{n}$. This completes the proof of the lemma.

Note that $r_{n}(z)=O\left(1 / z^{2}\right)$ as $z \rightarrow \infty$, so the quadratic differential $r_{n}(z) d z^{2}$ has at the infinite point a pole of the second order. If the boundary of domain $D$ contains $z=\infty$, then the maximal value in (12) can be obtained at this point (and accordingly, $\left(1-|\zeta|^{2}\right)^{2}\left|r_{n}(\zeta) \chi_{D}(\zeta)\right|$ can attain its maximum at $\left.\zeta=\chi_{D}(\infty)\right)$.

Lemma 2. Let $D$ be a simply connected domain on $\widehat{\mathbb{C}}$ with quasiconformal boundary $L$ and such that $\left\|S_{g_{D}}\right\|_{\mathbf{B}_{2}}<1 / 2$. There exists a constant $c_{0}>0$ such that for any rational function $r_{n}$ with poles of order two on $L$ of the form (3) and with

$$
\left\|r_{n}\right\|_{\mathbf{B}_{2}(D)}<c_{0}
$$


the boundary points of $D$ at which the maximal value in (12) is attained are substantial for extremal quasiconformal extensions of conformal immersions $f: D \rightarrow \widehat{\mathbb{C}}$ generated by the Schwarzian equation $S_{f}=r_{n}$ on $D$.

Proof. It is sufficient to prove the lemma for domains with boundaries containing $\infty$. We first consider the canonical case $D=\mathbb{H}$ for which a somewhat stronger result will be obtained.

The equation $S_{f}(z)=\varphi(z)$ defines the conformal immersion $f_{\varphi}: \mathbb{H} \rightarrow$ $\widehat{\mathbb{C}}$ determined uniquely by the requirement to preserve the points $0,1, \infty$.

By the Ahlfors-Weill theorem [1], every $\varphi \in \mathbf{B}_{2}(\mathbb{H})$ with $\|\varphi\|<1 / 2$ is the Schwarzian derivative $S_{f}$ of a univalent function $f$ in $\mathbb{H}$, and $f$ has a quasiconformal extension onto the lower half-plane $\mathbb{H}^{*}$ with Betrami coefficient of the form

$$
\mu_{\varphi}(z)=-2 y^{2} \varphi(\bar{z}), \quad \varphi=S_{f}(z=x+i y \in \mathbb{H})
$$

called the harmonic Beltrami coefficient (in the spirit of the KodairaSpencer deformation theory).

Our aim is to show that for every $r_{n}$ with real poles $a_{j}$ of order two and $\left\|r_{n}\right\|_{\mathbf{B}_{2}(\mathbb{H})}<1 / 2$ the corresponding harmonic Beltrami coefficient $\mu_{r_{n}}$ in $\mathbb{H}$ is extremal in its class, and

$$
\varkappa\left(f_{r_{n}} \circ \sigma\right)=k\left(f_{r_{n}}\right)=\left\|r_{n}\right\|_{\mathbf{B}_{2}\left(\mathbb{H}^{*}\right)},
$$

where $\sigma$ is the appropriate Moebius map of $\mathbb{D}^{*}$ onto $\mathbb{H}^{*}$.

It suffices to establish the relations (15) for $r_{n}$ with sufficiently small norm.

Pick the point $a_{0} \in \mathbb{R}$ at which the equality in (12) is attained, and two points $x^{\prime}, x^{\prime \prime}$ located on $\mathbb{R}$ in the left to all poles $a_{j}$ (so, $\mu_{r_{n}}(z)=0$ on $\left.\left[x^{\prime}, x^{\prime \prime}\right]\right)$. We now establish that

$$
\sup _{\|\psi\|_{A_{2}(\mathbb{H})}=1}\left|\left\langle\mu_{r_{n}}, \psi\right\rangle_{\mathbb{H}}\right|=\sup _{\|\psi\|_{A_{2}^{2}(\mathbb{H})}=1}\left|\left\langle\mu_{r_{n}}, \psi\right\rangle_{\mathbb{H}}\right|=H_{a_{0}}(f),
$$

which implies the equalities (15) and extremality of $\mu_{r_{n}}$ in its class.

Using the conformal map $z=g(\zeta)$ of the half-strip

$$
\Pi_{+}=\{\zeta=\xi+i \eta: \xi>0,0<\eta<1\}
$$

onto $\mathbb{H}$ with $g\left(x^{\prime}\right)=0, g\left(x^{\prime \prime}\right)=1, g\left(a_{0}\right)=\infty$, we pull-back $\mu_{r_{n}} / b\left(a_{0}\right)$ (where $b\left(a_{0}\right)$ is the local boundary dilatation at the point $a_{0}$ ) to the Beltrami coefficient

$$
\mu_{*}(\zeta):=\frac{1}{H_{a_{0}}} g_{*}\left(\mu_{r_{n}}\right)(\zeta)=\frac{1}{H_{a_{0}}}\left(\mu_{r_{n}} \circ g\right)(\zeta) \overline{g^{\prime}(\zeta)} / g^{\prime}(\zeta)
$$


on $\Pi_{+}$, which satisfies $\lim _{\xi \rightarrow \infty}\left|\mu_{*}(\xi+i \eta)\right|=\left\|\mu_{*}\right\|_{\infty}=1$ and has the limit function

$$
\mu_{*}\left(\zeta_{0}\right)=\lim _{\zeta \rightarrow \zeta_{0} \in \partial \Pi_{+}} \mu_{*}(\zeta)
$$

with

$$
\mu_{*}(i \eta)=0 .
$$

We claim that the sequence

$$
\omega_{m}(\zeta)=\frac{1}{m} e^{-\zeta / m}, \quad m=1,2, \ldots\left(\zeta \in \Pi_{+}\right),
$$

is degenerating for $\nu_{*}$. First of all, these $\omega_{m}$ belong to $A_{2}^{2}\left(\Pi_{+}\right) ; \omega_{m}(\zeta) \rightarrow$ 0 uniformly on $\Pi_{+} \cap\{|\zeta|<M\}$ for any $M<\infty$, and $\left\|\omega_{m}\right\|_{A_{2}\left(\Pi_{+}\right)}=1$. Further,

$$
\begin{aligned}
& \left\langle\mu_{*}, \omega_{m}\right\rangle_{\Pi_{+}}=\frac{1}{m} \iint_{\Pi_{+}} \mu_{*}(\zeta) \omega_{m}(\zeta) d \xi d \eta \\
= & \int_{0}^{1} e^{-i \eta / m} d \eta\left(\frac{1}{m} \int_{0}^{\infty} \mu_{*}(\xi+i \eta) e^{-\xi / m} d \xi\right) .
\end{aligned}
$$

The inner integral can be evaluated using the Laplace transform of $\mu_{*}$ in $\xi$. Integrating by parts and applying (17), one obtains

$$
\int_{0}^{\infty} \frac{\partial \mu_{*}(\xi+i \eta)}{\partial \xi} e^{-\xi / m} d \xi=\frac{1}{m} \int_{0}^{\infty} \mu_{*}(\xi+i \eta) e^{-\xi / m} d \xi
$$

On the other hand, Abel's theorem for the Laplace transform yields that the nontangential limit

$$
\lim _{s \rightarrow 0} \int_{0}^{\infty} \frac{\partial \mu_{*}(\xi+i \eta)}{\partial \xi} e^{-s \xi} d \xi=\int_{0}^{\infty} \frac{\partial \mu_{*}(\xi+i \eta)}{\partial \xi} d \xi=\mu_{*}(\infty)-\mu_{*}(i \eta) ;
$$

hence,

$$
\lim _{m \rightarrow \infty} \frac{1}{m} \int_{0}^{\infty} \mu_{*}(\xi+i \eta) e^{-\xi / m} d \xi=\mu_{*}(\infty) .
$$

By Lebesgue's theorem on dominated convergence, the iterated integral in (18) is estimated as follows

$$
\lim _{m \rightarrow \infty}\left|\left\langle\nu_{*}, \omega_{m}\right\rangle_{\Pi_{+}}\right|=\left|\int_{0}^{1} d \eta \lim _{m \rightarrow \infty} \frac{1}{m} \int_{0}^{\infty} \mu_{*}(\xi+i \eta) e^{-\xi / m} d \xi\right|=1 .
$$


Since by (12), the left-hand side equals to $\left\|\mu_{*}\right\|_{\infty}$ and all functions $\omega_{m}$ belong to $A_{2}^{2}\left(\Pi_{+}\right)$, this proves our claim.

Now, applying the inverse conformal map $\zeta=g^{-1}(z): \Pi_{+} \rightarrow \mathbb{H}$, one obtains the degenerating sequence

$$
\left\{\psi_{m}=\left(\omega_{m} \circ g^{-1}\right)\left(g^{\prime}\right)^{-2}\right\} \subset A_{2}^{2}(\mathbb{H}),
$$

for the initial Beltrami coefficient $\mu_{r_{m}}$ on $\mathbb{H}$. By (19),

$$
\lim _{m \rightarrow \infty}\left|\left\langle\mu_{r_{m}}, \psi_{m}\right\rangle_{\mathbb{H}}\right|=\left\|\mu_{r_{m}}\right\|_{\infty}=1
$$

which implies, together with Lemma 1, the assertion of Theorem 4 for the half-plane.

Let now $D$ be the generic simply connected domain bounded by quasicircle $L$ passing through $0,1, \infty$ and such that the Schwarzian of the conformal map $g_{D}$ of $\mathbb{H}^{*}$ onto $D$ preserving the points $0,1, \infty$ satisfies

$$
\left\|S_{g_{D}}\right\|_{\mathbf{B}_{2}\left(\mathbb{H}^{*}\right)}<1 / 2
$$

Given a rational function $r_{n}(z)$ of the form (3) with poles on $L$, we consider the univalent solution $w=f_{n}(z)$ of the equation

$$
S_{w}(z)=t r_{n}(z), \quad z \in D
$$

and its composition with $g_{D}$, taking $t>0$ so small that

$$
S_{f_{n} \circ g_{D}}=\left(S_{f} \circ g_{D}\right)\left(g_{D}^{\prime}\right)^{2}+S_{g_{D}}
$$

also satisfies

$$
\left\|S_{f_{n} \circ g_{D}}\right\|_{\mathbf{B}_{2}\left(\mathbb{H}^{*}\right)}<1 / 2 .
$$

The Beltrami coefficients of arbitrary quasiconformal extensions $\widehat{g}_{D}$ and $\widehat{f}_{n}$ of $g_{D}$ and $f_{n}$, respectively, across the boundaries of their domains to $\widehat{\mathbb{C}}$ are related by

$$
\mu_{\widehat{f}_{n}} \circ \widehat{g}_{D}=\mu_{\widehat{f}_{n} \circ \widehat{g}_{D}^{-1}} \circ \widehat{g}_{D}=\frac{\mu_{\widehat{f}_{n} \circ \widehat{g}_{D}}-\mu_{\widehat{g}_{D}}}{1-\bar{\mu}_{\widehat{g}_{D}}} \mu_{\widehat{f}_{n} \circ \widehat{g}_{D}} \frac{\partial_{\zeta} \widehat{g}_{D}}{\bar{\partial}_{\zeta} \widehat{g}_{D}} .
$$

In particular, using their Ahlfors-Weill extensions (14), one gets, in view of (20),

$$
\mu_{\widehat{f}_{n}} \circ \widehat{g}_{D}=-2 t \eta^{2} r_{n} \circ g_{D}(\bar{\eta}) \frac{\partial_{\zeta} \widehat{g}_{D}}{\partial_{\zeta} \widehat{g}_{D}}+O\left(t^{2}\right)=-2 t \lambda_{D}^{-2} r_{n}+O\left(t^{2}\right),
$$


or equivalently, for $z=g_{D}(\bar{\zeta})$,

$$
\mu_{\widehat{f}}(z)=-2 t \lambda_{D}^{-2}(z) r_{n}(z)+O\left(t^{2}\right) \quad \text { as } t \rightarrow 0 .
$$

The remainders in the last two equalities are uniformly bounded in $L_{\infty}$ norm for all $t$ for which the bound (21) is valid.

We establish now that the harmonic Beltrami coefficient

$$
\nu_{t r_{n}}(z)=t \lambda_{D}^{-2}(z) r_{n}(z)
$$

is infinitesimally extremal in its equivalence class.

Indeed, taking again the point $a_{0} \in L$ on which the upper limit (12) for chosen $r_{n}$ is attained and mapping the domain $D$ conformally onto the half-strip $\Pi_{+}$so that $a_{0}$ is going to $\infty$, one can repeat for $f_{n}$ the above arguments and derive from (16) and (19) that the boundary dilatation at $a_{0}$ is equal to $\left\|r_{n}\right\|_{\mathbf{B}_{2}(D)}$. This yields that the Beltrami coefficient (22) is infinitesimally extremal in $\operatorname{Belt}(D)_{1}$ and, moreover, its norm is attained on functions from $A_{2}^{2}(D)$.

As was mentioned in Section 4.1, such a Beltrami coefficient must be simultaneously globally extremal in its equivalence class. This implies the assertions of Lemma 2 and of Theorem 4, completing their proofs.

\section{References}

[1] L. V. Ahlfors, G. Weill, A uniqueness theorem for Beltrami equations // Proc. Amer. Math. Soc., 13 (1962), 975-978.

[2] A. Amboladze, H. Wallin, Rational interpolants with prescribed poles, theory and practice // Complex Var. Theory Appl., 34(4) (1997), 399-413.

[3] K. Astala, Selfsimilar zippers, Holomorphic Functions and Moduli, vol. I (D. Drasin et al., eds.), Springer-Verlag, New York, 1988, pp. 61-73.

[4] L. Bers, An approximation theorem // J. Anal. Math. 14 (1965), 1-4.

[5] L. Bers, A non-standard integral equation with applications to quasiconformal mappings // Acta Math., 116 (1966), 113-134.

[6] C. J. Earle, I. Kra, S. L. Krushkal, Holomorphic motions and Teichmüller spaces // Trans. Amer. Math. Soc., 944 (1994), 927-948.

[7] C. J. Earle, Zong Li, Isometrically embedded polydisks in infinite dimensional Teichmüller spaces // J. Geom. Anal., 9 (1999), 51-71.

[8] F. P. Gardiner, N. Lakic, Quasiconformal Teichmüller Theory // Amer. Math. Soc., 2000.

[9] G. M. Golusin, Geometric theory of Functions of a Complex Variable, Transl. of mathematical monographs, vol. 26, Transl. of Geometricheskaya teoriya funktcii kompleksnogo peremennogo, 2nd ed, Amer. Math. Soc., Providence, RI, 1969 . 
[10] H. Grunsky, Koeffizientenbedingungen für schlicht abbildende meromorphe Funktionen // Math. Z., 45 (1939), 29-61.

[11] A. Gustafsson, Approximation with rational interpolants in $A^{-\infty}(D) / /$ Comput. Methods Func. Theory, DOI 10.1007/s40315-016-0187-6.

[12] S. L. Krushkal, Quasiconformal Mappings and Riemann Surfaces, Wiley, New York, 1979.

[13] S. L. Krushkal, Grunsky coefficient inequalities, Carathéodory metric and extremal quasiconformsal mappings // Comment. Math. Helv., 64 (1989), 650660.

[14] S. L. Krushkal, Strengthened Moser's conjecture, geometry of Grunsky inequalities and Fredholm eigenvalues // Central European J. Math., 5(3) (2007), $551-580$.

[15] S. L. Krushkal, Rational approximation of holomorphic functions and geometry of Grunsky inequalities // Contemporary Mathematics, 455 (2008), 219-236.

[16] S. L. Krushkal, Strengthened Grunsky and Milin inequalities // Contemp. Math., 667 (2016), 159-179.

[17] R. Kühnau, Verzerrungssätze und Koeffizientenbedingungen vom Grunskyschen Typ für quasikonforme Abbildungen // Math. Nachr., 48 (1971), 77105.

[18] R. Kühnau, Wann sind die Grunskyschen Koeffizientenbedingungen hinreichend für Q-quasikonforme Fortsetzbarkeit? // Comment. Math. Helv., 61 (1986), 290-307.

[19] N. Lakic, Strebel Points // Lipa's Legacy, Contemporary Mathematics, 211, Amer. Math. Soc., Providence, RI, 2001, 417-431.

[20] I. M. Milin, Univalent Functions and Orthonormal Systems, Transl. of Mathematical Monographs, vol. 49, Transl. of Odnolistnye funktcii i normirovannie systemy, Amer. Math. Soc., Providence, RI, 1977.

[21] Z. Nehari, The Schwarzian derivative and schlicht functions // Bull. Amer. Math. Soc., 55 (1949), 545-551.

[22] Chr. Pommerenke, Univalent Functions, Vandenhoeck \& Ruprecht, Göttingen, 1975.

[23] K. Strebel, On the existence of extremal Teichmueller mappings // J. Anal. Math., 30 (1976), 464-480.

[24] W. P. Thurston, Zippers and univalent functions, The Bieberbach Conjecture: Proceedings of the Symposium on the Occasion of its Proof (A. Baernstein II et al., eds.), Amer. Math. Soc., Providence, R.I., 1986, 185-197.

[25] J. L. Walsh, Interpolation and Approximation by Rational Functions in the Complex Domain, 4th edn, Colloq. Publ., vol. XX, Amer. Math. Soc., Providence, RI, 1965. 
[26] L. Zalcman, Analytic Capacity and Rational Approximation, Lecture Notes in Math., 50, Springer, Berlin, 1968.

\section{CONTACT INFORMATION}

Samuel L. Krushkal Department of Mathematics, Bar-Ilan University, Israel,

Department of Mathematics, University of Virginia, Charlottesville, USA

E-Mail: krushkal@math.biu.ac.il 